\title{
CrossFit athletes exhibit high symmetry of fundamental movement patterns. A cross-sectional study
}

\author{
Silvio Tafuri ${ }^{1}$ \\ Angela Notarnicola ${ }^{2}$ \\ Antonello Monno ${ }^{2}$ \\ Francesco Ferretti ${ }^{2}$ \\ Biagio Moretti²
}
1 Department of Biomedical Science and Human On- cology, Aldo Moro University of Bari, Italy
2 Department of Orthopaedics, Aldo Moro University of Bari, Italy

Corresponding author:

Angela Notarnicola

Department of Orthopaedics, Aldo Moro University

of Bari

Piazza G. Cesare 11

70124 Bari, Italy

E-mail: angelanotarnicola@yahoo.it

\section{Summary}

Background: even if CrossFit training programs accounted actually more than 7500 gyms affiliated in the USA and more than 2000 in Europe and involved today more than 1 million of people, actually there were not several studies about the effect of the CrossFit on the health and sport performance. The aim of these research was to evaluate the performance in 7 fundamental movement patterns using a standardized methods, the Functional Movement Screen (FMS).

Methods: we enrolled three groups of athletes (age 17-40 years; $>6$ months of training programs): CrossFitters, body builders and professional weightlifters. FMS test was performed to all people enrolled. Scores of FMS test was examined comparing three groups.

Results: no differences in the three groups were showed in the mean score values of each test and in total score, except for shoulder mobility test (higher among CrossFitters) and trunk stability push-up test (higher among weightlifter). Agreement between the test performed on the two sides was higher in CrossFit groups for hurdle step $(93.2 \%)$, in line lung $(86 \%)$, rotary stability test $(95.3 \%)$ and shoulder mobility $(90.7 \%$; $p<0.001)$.

Conclusions: CrossFitters seem to have a high level of concordance in the scores achieved in bi- lateral test. CrossFit seems to produce marked symmetry in some fundamental movements compared to weightlifting and bodybuilding.

KEY WORDS: CrossFit, functional movement screen, mobility, training.

\section{Introduction}

CrossFit is a constantly varied, high intensity, functional movement strength and conditioning program which has seen a huge growth in popularity around the world since its inception twelve years ago ${ }^{1}$. Crossfit program consisted of lifts such as the squat, deadlift, clean, snatch, and overhead press performed as quickly as possible. Additionally, the CrossFit program included skill work for the improvement of traditional Olympic lifts and selected gymnastic exercises ${ }^{2,3}$.

There were not several studies about the effect of the CrossFit on the health and sport performance. A survey carried out on a little sample of healthy subjects seems to show that CrossFit program improved $\mathrm{VO}_{2}$ max and body composition in subjects of both genders across all levels of fitness ${ }^{2}$, but this paper has become involved in three different lawsuits ${ }^{4}$. A study performed in 2014 among 23 overweight adults seemed to show that subjects who practices CrossFit programs are less like to drop out exercise enjoyment but no change in BMI and body composition were found in these people ${ }^{5}$.

A 2012 review of the literature about the non-traditional training modalities, such as CrossFit, concluded that most of these forms of physical training are not supported in the scientific literature and required further investigations ${ }^{6}$. Despite these recommendation, in PubMed there were actually only 10 papers about the CrossFit program. Of these, 3 are case reports of very important injury during or after CrossFit Training, such as retinal detachment ${ }^{7}$, rhabdomyolisis $^{8}$, and cervical internal carotid artery dissection ${ }^{9}$. The relevance of these observation are very questionable and an epidemiological study seems to suggest that the incidence of injury in the CrossFit is similar with other sports ${ }^{1}$.

The research about effects of CrossFit on the fitness, wellness and health is currently poor (all the study carried out involved less than 100 subjects) and new study are needed to evaluate this form of physical training, that accounted more than 7500 gyms affiliated in the USA and more than 2000 in Europe and involved today more than 1 million of people ${ }^{3}$.

The main aim of the CrossFit program is to perform 
functional movements at high intensity and according to this view point, the Authors designed these research, with the aim to evaluate the performance in 7 fundamental movement patterns using a standardized methods.

\section{Materials and methods}

To evaluate the role of the CrossFit in conditioning the performance in fundamental movement, we designed an observational survey. The research was conducted according to international ethical standards ${ }^{13}$. We enrolled three groups of athletes:

1. "CrossFit Group": people who performed CrossFit program in affiliated boxes of the city of Bari, at least sixty months;

2. "body builders Group", people who attend a body building training program, at least sixty months;

3. "weightlifting group" professional weightlifters of Italian Federation of Weightlifting (FIPE), who performed this program from 6 months or more.

People who carried out two training programs at the time of the study or in the past 6 months were excluded.

In the design of the study, we choose as control group a traditional sport (weightlifting) and the most practiced sports for non professionals athletes (body building).

The research was carried out in the springer of 2015 , in the afternoon for all athletes; according to Italian standard, the temperature in the gym was of $19^{\circ} \mathrm{C}$.

The researchers illustrated to the enrolled people the aim of the study and informed, written consent were requested and obtained. For the participants aged $<18$ years, the consent was obtained by the parents.

The enrolled athletes were tested by Functional Movement Screen (FMS). FMS is a screening system that attempts allow the professional to assess the fundamental movement patterns of an individual. The FMS is comprised of seven fundamental movement patterns (tests) that require a balance of mobility and stability (including neuromuscular/motor control). These fundamental movement patterns are designed to provide observable performance of basic locomotor, manipulative, and stabilizing movements. The tests place the individual in extreme positions where weaknesses and imbalance become noticeable if appropriate stability and mobility is not utilized ${ }^{10,11}$.

FMS is composed of seven tests: deepsquat, hurdle step, in line lunge, shoulder mobility, active straight leg-rais, trunk stability push-up and rotary stability. The majority of the tests in the FMS examine both the right and left sides, and it is important that both sides are scored 10,11 .

The scoring for the FMS consists of four discrete possibilities. The scores range from zero to three, three being the best possible score. An individual is given a score of zero if at any time during the testing he has pain anywhere in the body. If the patient does not score a zero, a score of one is given if the person is unable to complete the movement pattern or is un- able to assume the position to perform the movement. A score of two is given if the person is able to complete the movement but must compensate in some way to perform the fundamental movement. A score of three is given if the person performs the movement correctly without any compensation, complying with standard movement expectations associated with each test. The best total score that can be attained on the FMS is twenty-one ${ }^{10,11 .}$

The FMS test was administered by two independent evaluators and score assigned by each evaluator was compared. If the score was different, we attributed the average score.

Sample size was set, using published data about FMS, on 20 subjects per groups.

Results of FMS test and inquiries about gender, age, sport practiced in the past (Yes/No) and musculoskeletal diseases were reported in standardized forms. Completed forms were inputted in a database created by FileMaker Pro and data were analysed by STATA MP11 software.

Continuous variables were described as means \pm standard deviations and ANOVA test was performed to compare means in the three groups. Categorical variables were described as percentages and percentages in the three groups were compared by chisquare test. For results of Hurdle step, In line lunge, Shoulder Mobility, Active Straight leg raise and Rotary stability we compared score of the right and left sides, to evaluate the Symmetry; this comparison was made by $\mathrm{K}$ agreement and $\mathrm{z}$-score test was carried out. For all test, significance was set at $p<0.05$.

\section{Results}

We enrolled and studied 90 persons, of which 64 $(71.1 \%)$ were female, with an average age of $27.9 \pm 0.8$ years: $43(47.8 \%)$ athletes of CrossFit group, 26 (28.9\%) professional weightlifters and 21 (28.9\%) bodybuilders. The three groups were homogeneous for mean age $(F=2.81 ; p=0.07)$, gender distribution (chi-square $=0.34 ; p=0.84) .20 \%(n=18)$ of the sample reported a musculoskeletal disease, with no difference between the three groups (chisquare $=3.32 ; p=0.19) ; 85.6 \%(n=77)$ of enrolled people practiced almost a sport in the past and this proportion was similar in the three groups (chisquare $=3.8 ; p=0.15$ ).

Table 1 showed the average scores for each test and each groups and the average total scores. Both for shoulder mobility test (left and right) both for trunk stability push-up test, the multivariate analysis excluded the role of gender, previous practiced sports and musculoskeletal disease as confounding factors ( $p>0.05)$.

For hurdle step, the agreement between the test performed on the two sides (right and left) resulted of $86.7 \%(z=5.86 ; p<0.0001)$, higher among CrossFit athletes $(93.2 \% ; z=3.2 ; p=0.0007)$ than among bodybuilders $(85.7 \% ; z=2.08 ; p=0.02)$ and weightlifters (76.9\%; $z=3.49 ; p=0.0002)$. 
Table 1. Average scores for test of FMS in CrossFit athletes, weightlifters and bodybuilders.

\begin{tabular}{|c|c|c|c|c|c|}
\hline Test & CrossFit & Weightlifter & Bodybuilders & $\mathbf{F}$ & $\mathbf{p}$ \\
\hline Deepsquat & $2.1 \pm 0.3$ & $2.1 \pm 0.4$ & $2 \pm 0$ & 1.49 & 0.23 \\
\hline Hurdlestep- left & $2.1 \pm 0.3$ & $2 \pm 0.3$ & $2.1 \pm 0.5$ & 0.72 & 0.49 \\
\hline Hurdlestep- right & $2 \pm 0.4$ & $2 \pm 0.2$ & $2 \pm 0.4$ & 0.13 & 0.90 \\
\hline Inlinelunge- left & $2.2 \pm 0.4$ & $2.2 \pm 0.4$ & $2.2 \pm 0.4$ & 0.10 & 0.90 \\
\hline Inlinelunge- right & $2.1 \pm 0.5$ & $2.1 \pm 0.6$ & $2.1 \pm 0.5$ & 0.05 & 0.95 \\
\hline Shoulder mobility- left & $2.7 \pm 0.6$ & $2.2 \pm 1$ & $2.6 \pm 0.6$ & 3.93 & 0.02 \\
\hline Shoulder mobility- right & $2.8 \pm 0.6$ & $2.3 \pm 1$ & $2.8 \pm 0.4$ & 5.03 & 0.01 \\
\hline Activestraight leg raise- left & $2.6 \pm 0.7$ & $2.5 \pm 0.8$ & $2.5 \pm 0.7$ & 0.23 & 0.79 \\
\hline Activestraight leg raise- left & $2.5 \pm 0.6$ & $2.6 \pm 0.6$ & $2.4 \pm 0.7$ & 0.49 & 0.61 \\
\hline Rotary stability- left & $1.9 \pm 0.2$ & $1.9 \pm 0.3$ & $1.8 \pm 0.4$ & 1.24 & 0.29 \\
\hline Rotary stability- right & $1.9 \pm 0.3$ & $2 \pm 0.2$ & $1.9 \pm 0.3$ & 0.3 & 0.74 \\
\hline Trunk Stability Push-up & $2.0 \pm 0.8$ & $2.3 \pm 0.8$ & $1.6 \pm 0.8$ & 3.92 & 0.02 \\
\hline Total & $15.2 \pm 1.7$ & $14.8 \pm 2$ & $14.2 \pm 1.9$ & 2.28 & 0.10 \\
\hline
\end{tabular}

For in line lung, the agreement was $80 \%(z=5.63$; $\mathrm{p}<0.0001): 86 \%(z=4.5 ; \mathrm{p}<0.0001)$ for CrossFit group, $76.9 \%(z=3.24 ; p=0.0006)$ for bodybuilders and $71.4 \%(z=1.47 ; p=0.07)$ for weightlifters.

Shoulder mobility agreement achieved $81.1 \% \quad(z=7.0$; $\mathrm{p}<0.0001)$ and also for this test it was higher among CrossFitters $(90.7 \% ; z=5.5 ; p<0.0001)$ than among weightlifters $(84.6 \% ; z=5.5 ; p<0.0001)$ and bodybuilders $(57.1 \% ; z=-0.05 ; p=0.52)$.

Agreement for Active straight leg raise test was $77.8 \%(z=7.2 ; p<0.0001)$ and it was higher among weightlifters $(84.6 \% ; z=4.8 ; p<0.0001)$ than among bodybuilders $(76.2 \% ; z=3.44 ; p=0.003)$ and CrossFit group $(74.4 \% ; z=4.68 ; p<0.0001)$.

Finally, for Rotary stability test, the overall agreement between the two sides was of $92.2 \% \quad(z=4.79$; $p<0.0001), 95.3 \%(z=4.2 ; p<0.0001)$ in CrossFit group, $88.5 \%(z=0.29 ; p=0.61)$ among weightlifters and $90.5 \%(z=3.1 ; p=0.001)$ among bodybuilders.

\section{Discussion}

Our study showed that there were no differences in the performance of fundamental movement patterns between athletes trained in CrossFit programs and traditional programs, such as weightlifting or bodybuilding.
To correctly read this results, we must consider that this test was not designed to measure sport performance, but to screen fundamental movement competency, to identify deficient areas of mobility and stability and finally to prevent injuries ${ }^{12,13}$. In fact, some study showed that a FMS score $<11$ is associated with higher risk of injury ${ }^{14}$, even if this assumption is quite questioned ${ }^{15}, 16$.

Infact, an important factor in prevention of injuries and improving performance is to quickly identify deficits in symmetry, mobility, and stability because of their influences on creating altered motor programs throughout the kinetic chain ${ }^{17-19}$.

According to our results, the risk of injury for CrossFit athletes (evaluated by FMS) was similar to other training programs and it is consistent with the only epidemiologic investigation available ${ }^{1}$.

The mean score of our group seems similar (perhaps slowly higher) to other athletes, such as runners ${ }^{20}$ or division I college athletes ${ }^{21}$.

The main strength of this study was the homogeneity of the three groups, that were composed by athletes with a long history of training; unusually, the major part of the sample was composed by females. Another strength was the use of a validated, standardized test. The main weakness was the impossibility to make a pre and post analysis, that could require the enrolment of adolescents or children; this could be very difficult. 
CrossFit showed an higher score than weightlifting in the shoulder mobility test. This test assess bilateral and reciprocal shoulder range of motion, combining internal rotation with adduction of one shoulder and external rotation with abduction of the other. The test requires normal scapular mobility and thoracic spine extension. Poor performance during this test can be the result of several causes, one of which is the widely accepted explanation that increased external rotation is gained at the expense of internal rotation in overhead throwing athletes. In addition, excessive development and shortening of the pectoralis minor or latissimus dorsi muscles can cause postural alterations including rounded or forward shoulders ${ }^{15}$. This could partially explain the lower scores achieved for weightlifters.

Although, weightlifter showed an higher score for the trunk stability push-up. The trunk stability push-up tests the ability to stabilize the core and spine in an anterior and posterior plane during a closed-chain upper body movement. The test assesses trunk stability in the sagittal plane while a symmetrical upper extremity push-up motion is performed. The performance in this test is very notable, because, if the trunk does not have adequate stability during sport activities, kinetic energy will be dispersed and lead to poor functional performance, as well as the potential for micro traumatic injury ${ }^{15}$.

CrossFitters seem to have a high level of concordance in the scores achieved in bilateral test. CrossFit seems to produce marked symmetry in some fundamental movements compared to weightlifting (with the exception of active straight leg raise) and bodybuilding. This observation was not previously reported in the literature and must be studied in depth in future studies, also for its potential application for the athletes' health and for the role of the CrossFit as potential complementary discipline of the sports that needed basic athletic training.

\section{Conflict of interests}

The Authors declare that they have no conflict of interests regarding the publication of this paper.

\section{References}

1. Hak PT, Hodzovic E, Hickey B. The nature and prevalence of injury during CrossFit training. J Strength Cond Res. 2013.

2. Smith MM, Sommer AJ, Starkoff BE, Devor ST. Crossfit-based high-intensity power training improves maximal aerobic fitness and body composition. J Strength Cond Res. 2013;27 (11): 3159-3172.

3. CrossFit. What is CrossFit? http://www.crossfit.com/cfinfo/what-is-crossfit.html

4. Oransky I. CrossFit gym owner sues Ohio State, says fraudu- lent data led to $\$ 273$ million in NIH grants. Retraction Watch. http://retractionwatch.com/2015/05/08/crossfit-gym-ownersues-ohio-state-says-fraudulent-data-led-to-273-million-innih-grants/

5. Heinrich KM, Patel PM, O’Neal JL, Heinrich BS. High-intensity compared to moderate-intensity training for exercise initiation, enjoyment, adherence, and intentions: an intervention study. BMC Public Health. 2014;14:789.

6. O'Hara RB, Serres J, Traver KL, Wright B, Vojta C, Eveland E. The influence of nontraditional training modalities on physical performance: review of the literature. Aviat Space Environ Med. 2012;83(10):985-990.

7. Joondeph SA, Joondeph BC. Retinal Detachment due to CrossFit Training Injury. Case Rep Ophthalmol Med. 2013; 2013:189837.

8. Larsen C, Jensen MP. Rhabdomyolysis in a well-trained woman after unusually intense exercise. Ugeskr Laeger. 2014;176(25).

9. Lu A, Shen P, Lee $P$, et al. CrossFit-related cervical internal carotid artery dissection. Emerg Radiol. 2015;22(4):449-452.

10. Padulo J, Oliva F, Frizziero A, Maffulli N. Muscles, Ligaments and Tendons Journal. Basic principles and recommendations in clinical and field science. MLTJ. 2013;3(4):250-252.

11. Cook G, Burton L, Hoogenboom BJ, Voight M. Functional movement screening: the use of fundamental movements as an assessment of function - part 1. Int J Sports Phys Ther. 2014;9(3):396-409.

12. Cook G, Burton L, Hoogenboom BJ, Voight M. Functional movement screening: the use of fundamental movements as an assessment of function - part 2. Int $\mathrm{J}$ Sports Phys Ther. 2014;9(4):549-563.

13. Cook EG, Burton L, Hoogenboom BJ. The use of fundamental movements as an assessment of function-Part 1. N Am J Sports Phys Ther. 2006;1(2):62-72.

14. Cook EG, Burton L, Hoogenboom BJ. The use of fundamental movements as an assessment of function-Part 2. N Am J Sports Phys Ther. 2006;1(3):132-139.

15. Knapik JJ, Cosio-Lima LM, Reynolds KL, Shumway RS. Efficacy of functional movement screening for predicting injuries in coast guard cadets. J Strength Cond Res. 2015;29(5):11571162.

16. Engquist KD, Smith CA, Chimera NJ, Warren M. Performance Comparison of Student-Athletes and General College Students on the Functional Movement Screen and the Y Balance Test. J Strength Cond Res. 2015;29(8):2296-2303.

17. Kibler WB, Chandler TJ, Uhl T, Maddux RE. A musculoskeletal approach to the preparticipation physical examination: Preventing injury and improving performance. Am J Sports Med. 1989:17:525-527.

18. Nadler SF, Malanga GA, Feinberg JH, et al. Relationship between hip muscle imbalance and occurrence of low back pain in collegiate athletes: a prospective study. Am J Phys Med Rehabil. 2001;80:572-577.

19. Battie MC, Bigos SJ, Fisher LD, et al. Isometric lifting strength as a predictor of industrial back pain reports. Spine. 1989; 14:851-856.

20. Hotta T, Nishiguchi S, Fukutani N, et al. Functional Movement Screen for Predicting Running Injuries in 18-24 Year-Old Competitive Male Runners. J Strength Cond Res. 2015.

21. Warren M, Smith CA, Chimera NJ. Association of the Functional Movement Screen with injuries in division I athletes. J Sport Rehabil. 2015;24(2):163-170. 